\title{
Reading and Connecting: Using Social Annotation in Online Classes
}

\author{
Xinran Zhu ${ }^{1}$, Bodong Chen ${ }^{1, *}$, Rukmini Manasa Avadhanam ${ }^{1}$, Hong Shui ${ }^{1}$, and Raymond \\ Zhang $^{1}$
}

${ }^{1}$ University of Minnesota, Minneapolis, USA

*e-mail: chenbd@umn.edu

\begin{abstract}
The COVID-19 pandemic has forced many instructors to rapidly shift to online/distance teaching. With a narrow preparation window, many instructors are at a loss of strategies that are both effective in responding to the crisis and compatible with their professional practices. This article presents a systematic literature review of the use of web annotation to support reading and discussion activities in formal education. As a genre of information systems, web annotation allows users to socially engage with web resources and is used in education to support social reading, argumentation, group inquiry, and community building. From this review we identified five types of social annotation activity design: processing domain-specific knowledge; supporting argumentation and inquiry; improving literacy skills; supporting instructor or peer assessment, and connecting online learning spaces. Practical recommendations for incorporating social annotation in online/distance classes are provided.
\end{abstract}

Keywords: social annotation; web annotation; social reading; collaborative reading

Note: This is a preprint. Citation in the APA style: Zhu, X., Chen, B., Avadhanam, R., Shui, H., \& Zhang, R. (2020, May 9). Reading and Connecting: Using Social Annotation in Online Classes. https://doi.org/10.35542/osf.io/2nmxp.

\section{Introduction}

COVID-19 has forced schools and universities to pivot to online/distance learning. For many educators, this transition is an abrupt, emergent response to a crisis. While online/distance instruction does allow many learning activities to continue, attempts to fully replicate face-to-face (F2F) instruction online is misguided and misinformed, and are destined to miscarry. Instructors, especially those who have not taught online, are at a loss of teaching strategies that effectively integrate their professional practices and identities in online teaching environments.

Among various instructional needs (such as online videoconferencing and proctoring), one learning activity that is popular in classrooms at all educational levels is the social reading of course materials. No matter whether the learning objective is to grasp scientific concepts or to critique a literary text, it is a common classroom practice to engage students in reading and discussing a shared document. This practice of discussing readings is supported by social constructivist views of learning that recognize critical roles played by language and social interaction in human development (Vygotsky, 1962). When facilitating student discussions in a F2F classroom, the teacher can rely on a range of verbal and non-verbal cues to diagnose student understanding, promote peer sharing, orchestrate turn-taking, and facilitate transactive knowledge construction (Chi and Menekse, 2015; Michaels et al., 2008). Moving online, teachers face a lingering question: What online teaching strategies are available to support social reading and group discussion of course materials across disciplines and educational levels?

To answer this question, we present a systematic literature review of the use of web annotation to support social reading in K-12 and higher education. Web annotation is a genre of information technology that allows a user to annotate information in a web document and anchor a discussion in the annotated information. As a matter of fact, annotation-paper-based or online-is an important part of human cognition; it serves a multitude of functions including procedural signals, placemarks, an interpretive activity, and a visible trace of a reader's attention (Marshall, 1997; O'Hara and Sellen, 1997). Since the pioneering Annotea project, web annotation tools have been created to support the annotation of web materials as well as social interaction around these materials (Haslhofer et al., 2012). In comparison with paper-based annotation, web annotation is not constrained by the need to simultaneously access a shared document and is posited to create a layer of interactivity on top of any web document (W3C Web Annotation Working Group, 2016). In education, web annotation is broadly used to support social reading, group sensemaking, knowledge construction, and community building (e.g., Chen, 2019; Kalir et al., 2020; Marshall, 1997; Plevinski et al., 2017). In the following sections, we first describe methodological details of the literature review, followed by a presentation of key findings. We conclude this review by offering practical recommendations that are applicable to the current COVID-19 situation and beyond. 


\section{Methods}

\section{Literature Search Strategy}

In April 2020, we conducted a comprehensive literature search using two search databases: EBSCO and Scopus. Scopus was eventually chosen because it yielded a broader set of results. On Scopus, the search was limited to Social Sciences to identify educational literature. To capture literature related to social annotation, we used an advanced combination of search terms including web/online annotation, social reading/annotation, collaborative reading/annotation, and anchored discussion; the asterisk character $(*)$ was used to match broad results (e.g., using "web* annotat*" to match both web and web-based annotation). The query was set to match studies in which these terms appear in the title, keywords, or abstract. Only referred journal articles were included. This initial search yielded 249 results.

\section{Inclusion Criteria}

We screened these studies based on the following inclusion criteria: (a) being about social or web annotation in online or web-based settings; (b) including empirical evidence of learning (rather than technology acceptance); (c) being published in electronic format and accessible online; (d) being written in English; (e) not being a literature review. Thirty-nine studies met the criteria and were included in this study for analysis (see Table 1 for an overview).

\section{Coding and Synthesis}

After the screening, five researchers read and extracted the following information from each article: level of education, subject area, learning theory, learning activity design, web annotation technology, research methods, and learning outcomes. After coding, studies were further categorized by the genre of learning activity design, resulting in five categories that are used to organize findings to be presented in the next section.

\section{Using Web Annotation in Classrooms}

\section{Processing Domain-Specific Knowledge}

Web annotation is extensively used to support learner sensemaking of domain-specific knowledge in both K-12 and higher education. With web annotation tools, learners can annotate online texts, discuss and interact with each other, and connect the texts with their prior knowledge for deeper understanding. For instance, Mohn Nor, Hazman, and Hamat (2013) designed an online reading system called The Annotation Tool to facilitate reading processes for ESL (English as a Second Language) learners in an undergraduate class in Malaysia. To facilitate language acquisition and domain understanding, the instructors provided guiding questions and prompts for students' group annotation activities. Results from student questionnaires and interviews indicated that web annotations were useful for students to organize their thinking, identify main ideas, and summarize key concepts.

In a study involving medical pathology junior and senior college students, individual and collaborative annotation activities were designed to help students differentiate the images of normal and abnormal human tissues (Sahota et al., 2016). Relying on a Virtual Microscopy technology and an image annotation tool called Slice, students viewed and annotated digitized versions of microscopic slides, followed by quizzes on the annotated topic, questionnaires and instructor feedback. For senior students, research findings showed a significant improvement in students' quiz scores and understanding of the topics following the collaborative annotation activity, as compared to the quiz after individual annotation activity. This study also found that collaborative annotations bolstered learning outcomes, interactivity, and engagement for senior students, compared to junior students, who had less prior knowledge, and collaboration with each other.

In an undergraduate physics class, a "seeded annotation" intervention was designed to enhance knowledge acquisition by embedding high-quality annotations made by former cohorts in the materials read by the current class (Miller et al., 2016). Findings revealed a significant positive effect of this intervention on both the quality and quantity of student annotations, as well as more prompt participation by students. Collaborative annotation using the WCRAS-TQAFM tool with mechanisms filtering poor quality annotations promoted reading performance and reduced the cognitive load of high school students in Taiwan (Jan et al., 2016). Other studies (Zarzour and Sellami, 2017, 2018) also discussed the impact of social annotation on learner achievement in undergraduate computer science courses. Overall, social annotation is popularly used in classrooms to support student engagement with domain-specific materials. 


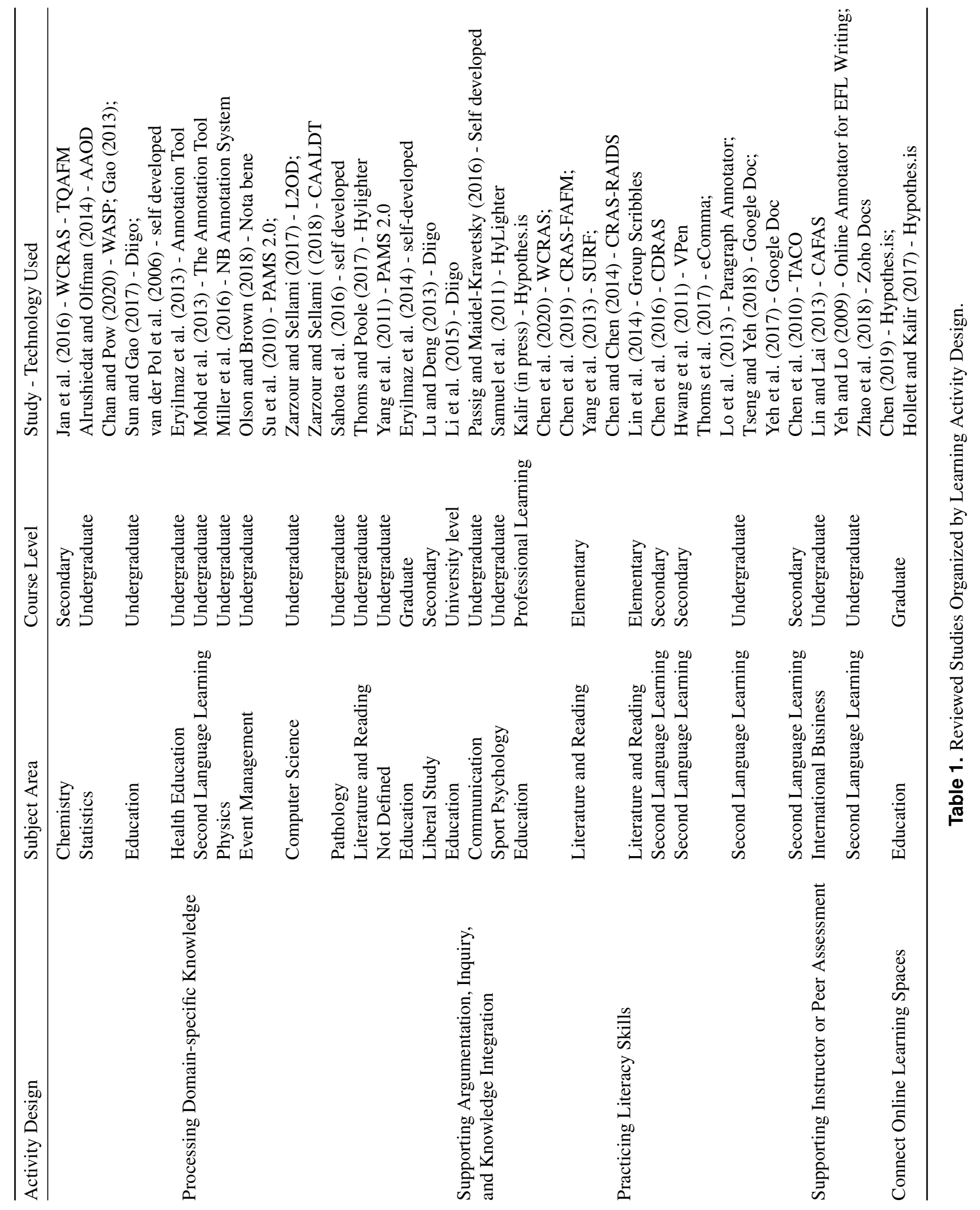




\section{Supporting Argumentation, Inquiry, and Knowledge Construction}

While domain understanding can be facilitated by annotating materials, in some cases the generated annotations are further aggregated to construct arguments, support inquiry, and integrate multiple perspectives.

In a study by Lu and Deng (2013), Diigo, a social bookmarking and web annotation tool, was incorporated into one Liberal Study module to help high-school students critically read source materials, evaluate multiple viewpoints, and build arguments about "whether Hong Kong should construct a high-speed rail system." To these ends, students first highlighted key concepts (in green), supportive views (red), and opposing views (blue) in articles selected beforehand by the instructor. For each highlight, students could further add sticky notes to present evidence using argumentative tags ("supportive position" or "opposing position"). The annotations were extracted from Diigo for group members to discuss, negotiate, and produce well-reasoned arguments. Research findings showed social annotation supported students to become more attentive, critical, and reflective in the process of developing claims.

In another study that also adopted Diigo, Li, Pow and Cheung (2015) engaged university students in a collaborative inquiry into authentic, complex, ill-structured social issues (e.g., "Should the right to public knowledge override the right to privacy or vice versa?"). Without any assigned materials, group members searched and bookmarked web pages to build a Diigo database around their selected social issue. Their Diigo annotations, comprising highlighted text snippets, annotations, and responses, were fed into a group deliberation to co-create mind maps about the social issue with supporting facts and evidence. During the collaborative inquiry, learners were found to engage in meaning negotiations and knowledge co-construction. The instructor provided facilitation mainly in the initializing stage (e.g., offering feedback on problem selection) and intervened in the following stages only when necessary.

Together with other studies (Chan and Pow, 2020), the literature shows social annotation provides unique affordances for students to organize information and ideas to support argumentation, collaborative inquiry, and knowledge construction.

\section{Improving Literacy Skills}

Social annotation can not only be used as a knowledge processing and application tool, it can also support literacy skills to promote reading comprehension and language capabilities. This is especially useful in first and second language learning classrooms where reading plays a key role in achieving language learning objectives (Hwang et al., 2011; Lin et al., 2014). In this case, the reading content becomes less important than the language skills that are transferable across subject domains.

In an undergraduate English Reading class, 22 low-achieving EFL (English as a Foreign Language) learners participated in an annotation and discussion activity within a group of 3 or 4 using the commenting feature in Google Docs (Tseng and Yeh, 2018). Following the Reciprocal Teaching approach (Palincsar and Brown, 1984), students applied four group discussion strategies in the activity: predicting, questioning, clarifying, and summarizing. Results showed significant improvement in reading comprehension skills among students. Students perceived questioning and predicting as the most useful strategies, which had initiated their collaborative reading and led to a transformation from passive reading to a deeper level of comprehension. The results corroborated previous research (Yeh et al., 2017) suggesting deeper reading comprehension was triggered by generating and responding to questions.

To improve elementary students' reading performance, Chen and Chen (2014) devised a social annotation and scaffold system named CRAS-RAIDS. To help students create structured and meaningful annotations, the system provided seven reading scaffolds (e.g., "reasoning" and "discrimination") and six discussion scaffolds (e.g., "quizzing" and "clarifying"). With the provided cognitive and metacognitive scaffolding, students did not only improve in direct comprehension in "shallow" reading but also promoted inferential comprehension in deep reading. Importantly, students' reading strategies had been enhanced by integrating metacognition in the reading and annotation process.

Along with other studies (Lo et al., 2013; Thoms et al., 2017; Yang et al., 2013), the social annotation has shown efficacy in promoting productive discussions and literacy skills. Through annotating, students revisit ideas in the reading, identify reading structures, engage in dynamic social interaction, and develop higher-level cognitive abilities. Finally, the literacy skills they gain can be potentially transferred into other domains.

\section{Supporting Instructor or Peer Assessment}

Web annotations allow for an additional opportunity for assessment and peer/instructor feedback that is conducive to improved learning outcomes and engagement (Lin and Lai, 2013).

In the simplest form, an instructor can provide feedback by leaving annotations in online documents submitted by EFL college students to help them correct writing errors (Yeh and Lo, 2009). In a more sophisticated technological setup, Chen, Chen, and Sun (2010) studied whether an algorithmically generated scoring mechanism that was integrated into the Tag-based Collaborative Reading Learning (TACO) system could increase instructor evaluation accuracy and support students' reading comprehension. Using the Vector Space Model (VSM), WordNet, Latent Semantic Analysis (LSA), and summarization algorithms, the tag-based scoring mechanism analyzes student-generated annotations and produces a score about each student's progression on reading comprehension. This scoring mechanism allows 56 EFL high school students to gauge how good their 
tags were, re-read the article if their original tags received low scores, and discuss tags with their peers. The use of social tagging along with algorithmic scoring was received well by students and instructors and led to improved reading comprehension skills as well as the instructor's enhanced capacity to assess progress.

Another study situated in an undergraduate class in Taiwan, where researchers Lin and Lai (2013) studied the use of social annotation as a formative assessment and feedback mechanism. Using the Collaborative Annotation Formative Assessment System (CAFAS), students received feedback both from instructors as well as from a database of peer generated social annotations. When reviewing an incorrectly answered question, the student could use existing annotations to rectify misconceptions; in case no meaningful annotations were provided, the student could seek additional sources to understand the error and share back to the database via annotations. Students benefited from the system because they were willing to spend more time on review and collaborative annotation because it is a learning aid that is convenient and produces a stable environment. Instructors may also use annotations to diagnose student learning for teaching adjustments.

In summary, the integration of a feedback process into social annotation systems may allow students to put more effort into, create a more engaging environment, devote more time to review and improve foreign language learning.

\section{Connecting Online Learning Spaces}

Due to its networked qualities, web annotation can be used to help learners cross boundaries between learning spaces (e.g., formal and informal) to make learning experiences more integrated, enjoyable, and personally meaningful. This use is especially relevant to adult learners and learning professionals who bring professional identities and social networks as they embark on a learning journey. For this audience, web annotation, together with many tools available in their learning ecologies, create and sustain learners' connections with people, resources, and ideas in the openly networked world.

Reflecting this use of web annotation, Hollett and Kalir (2017) designed "playgrids" that integrate web annotation and other social media tools to engage learners in professional learning. Playgrids, defined as "as the creative knitting together of social media tools to effectively participate across space, time, and scale" (Hollett and Kalir, 2017: 237), emphasizes play, an activity that involves movement within and across seemingly rigid structures. In one case of a Master's level course involving thirteen learners who were mostly working professionals, learners used Hypothes.is to support three types of playgrids: annotation across platforms, annotation as blog commentary, and annotation flash mobs. In an annotation flash mob, learners used Hypothes.is to create self-organized and improvisational dialogues beyond the course requirements. In this case, following a public invitation on Twitter and blogs, learners gathered at an online location at a particular time to annotate simultaneously. The synchronous annotation activities "spilled over" to other social media spaces like Twitter and reached learners' personal networks beyond the class.

In the case study by Chen (2019), the instructor intentionally decoupled learning from traditional learning management systems (LMS) to facilitate networked collaborative discussion by integrating web annotation and team communication technologies. In a graduate-level class, the instructor used Hypothes.is to engage students in annotating course readings on a weekly basis. Different from earlier examples, the instructor intentionally attempted to orchestrate idea movements across discourse spaces, provide multiple entry points to a common discussion, and make it easier for learners to cross boundaries and discourse spaces. Hypothes.is annotations were synchronized in the class' communication environment Slack, allowing learners to enter the discussion from different places. Research findings indicated that students formed dense social ties on both Hypothes.is and Slack; students also found Hypothes.is useful for collaborative sensemaking of challenging readings and community building. However, some earlier-starter students communicated challenges with the timing of participation; after leaving their annotations each week, they needed to go back to read the other students' contributions one week later.

In summary, social annotation does not only provide opportunities to deeply engage with web materials or inquiry activities, but it is also poised to serve as an infrastructure to connect online learning spaces to make learning experiences more connected, fun, and personally relevant.

\section{Practical Recommendations}

In this review, we synthesized the research on social annotation in formal classrooms into five areas of application (see Table I for a summary). Below, we offer four practical recommendations that are grounded in the review and cut across these five areas.

\section{Articulate Pedagogical Goals When Incorporating Social Annotation}

The findings of the literature review clearly indicate that the annotation strategies should be designed to meet the pedagogical intentions. As shown in this review, social annotation can be applied in many ways to support online teaching and learning. To generate desirable learning outcomes, it is important to clearly identify the pedagogical goals and align social annotation activities with these goals. For instance, in one study, social annotation is recommended over the Blackboard discussion forum if the pedagogical intention is to make "targeted critiques or self-reflections to specific sections of the material" rather than 
to post a long summary (Sun and Gao, 2017: 77). In another study that aimed to promote student argumentation, the social annotation was a means to support the process of building arguments from web sources (Li et al., 2015).

\section{Select Web Annotation Tools That Are Appropriate for the Context}

A wide range of web annotation technologies is represented in this review. Some tools are custom-built, with sophisticated functionalities that provide unique scaffolding (Chen and Chen, 2014; Eryilmaz et al., 2013). However, there are also studies that adopt tools that are readily available, such as Diigo, Hypothesis, and even Google Docs. This review provides an overview of key features of web annotation tools including highlighting, tagging, color-coding, filtering, and peer/annotation recommendation. While these features are not available in all web annotation tools, it is important to remember they are only useful when they serve pedagogical goals. When a custom feature such as guiding student attention by altering the font size (Eryilmaz et al., 2013) is not available, the instructor could be creative and pre-annotate the reading to provide similar attention guidance. General-purpose tools like Google Docs have advantages due to their low barriers to entry.

\section{Decide the Level of Instructor Involvement}

When it comes to the instructor involvement, more is not always better. The decision should take into consideration the learners' characteristics and the nature of the learning tasks. From the literature, most of the studies provided tool tutorials for students. Other than that, some activities were free-form and instructors only provided support when technical issues or discussion challenges emerged (Chen and Chen, 2014; Chen et al., 2019). This level of instructor involvement does not only save instructor time but also provides more flexibility and invites learners to contribute their perspectives.

In the meantime, several studies highlighted the need for scaffoldings in the technical, social, and content aspects especially for young and novice learners (Chen and Pow, 2020; Tseng and Yeh, 2018). For instance, some students perceived their reading being impeded and distracted by irrelevant annotations. To further scaffold social annotation, instructors can choose to provide prompt questions, reading strategies, and attention guidance (Eryilmaz et al., 2014; Sun and Gao, 2017; Tseng and Yeh, 2018). Additionally, several studies suggested that dividing the class into smaller groups and pairing low- and high-performing students would also enhance discussion quality and interaction (Chen et al., 2019; Thoms et al., 2017). Hollett and Kalir (2017) and Chen (2019) highlighted the possibility of annotating "in the open” and engaging learning professionals' personal social media networks. A deliberately designed involvement plan can avoid unnecessary efforts for both the instructors and students and therefore promote learning.

\section{Develop An Evaluation Strategy}

As demonstrated in several studies, social annotation is not for everyone and there are emerging challenges. For instance, not all annotation tools were found intuitive to use, as found in a study involving graduate students (Chen, 2019). In another study, undergraduate students reported difficulty in making distinct annotations, while some peer annotations might even impede their understanding (Thoms and Poole, 2017). Therefore, it is important to create a feedback loop and a strategy to evaluate the use of social annotation in a particular context. Such an evaluation strategy could cover a range of areas including targeted learning outcomes, technology usability, inclusivity, and socio-emotional factors.

As web annotation activities generate digital trace data that provide indicators of learning, another approach is to derive learning analytics that extracts annotation data to be analyzed for feedback purposes. For instance, the Crowd Layers is a dashboard tool that extracts data from Hypothes.is and reports learner engagement measures to be used by learners and educators (Kalir, in press). Chen, Chen, and Sun (2010) used learner annotation data to assess domain understanding. Social network analysis of annotation data is used by Chen (2019) to examine peer interaction patterns. Web annotation data can be harnessed to assess learning and evaluate the extent to which pedagogical goals are attained by incorporating social annotation.

\section{Conclusion}

In the COVID-19 pandemic, we need to forge and sustain connections more than ever. While video conferencing provides one way to connect learners from distance in a synchronous manner, this review suggests unique affordances web annotation technologies could provide for online learning to achieve various pedagogical goals. In particular, web annotation can help processing domain-specific knowledge; supporting argumentation, inquiry, and knowledge construction; improving literacy skills; supporting instructor or peer assessment, and connecting online learning spaces. It is our hope that these identified application areas, in combination with four practical recommendations, would provide pragmatic and evidence-based support for educators to engage learners in reading, learning, and connecting.

\section{References}

Alrushiedat, N. and Olfman, L. (2014), “Anchoring for self-efficacy and success”, in 2014 47th Hawaii International Conference on System Sciences, IEEE, pp. 13-21. 
Chan, J.W.W. and Pow, J.W.C. (2020), "The role of social annotation in facilitating collaborative inquiry-based learning", Computers \& Education, Vol. 147, pp. 1-13.

Chen, B. (2019), "Designing for networked collaborative discourse: An UnLMS approach”, TechTrends, Springer, Vol. 63 No. 2, pp. 194-201.

Chen, C.M. and Chen, F.Y. (2014), "Enhancing digital reading performance with a collaborative reading annotation system", Computers \& Education, Vol. 77, pp. 67-81.

Chen, C.M., Chen, L.C. and Horng, W. J. (2019), “A collaborative reading annotation system with formative assessment and feedback mechanisms to promote digital reading performance", Interactive Learning Environments, Taylor \& Francis, pp. $1-18$.

Chen, C.M., Li, M.C. and Chen, T.C. (2020), “A web-based collaborative reading annotation system with gamification mechanisms to improve reading performance", Computers and Education, Vol. 144, pp. 1-17.

Chen, C.M., Wang, J.Y., Chen, Y.T. and Wu, J.H. (2016), "Forecasting reading anxiety for promoting English-language reading performance based on reading annotation behavior", Interactive Learning Environments, Vol. 24 No. 4, pp. $681-705$.

Chen, J.M., Chen, M.C. and Sun, Y.S. (2010), “A novel approach for enhancing student reading comprehension and assisting teacher assessment of literacy”, Computers \& Education, Elsevier, Vol. 55 No. 3, pp. 1367-1382.

Chi, M. T. H., and Menekse, M. (2015), “Dialogue patterns that promote learning”, in L. B. Resnick, C. Asterhan \& S. N. Clarke (ed.), Socializing intelligence through academic talk and dialogue, AERA, Washington, DC, pp. $263-274$.

Eryilmaz, E., Chiu, M.M., Thoms, B., Mary, J. and Kim, R. (2014), "Design and evaluation of instructor-based and peer-oriented attention guidance functionalities in an open source anchored discussion system", Computers \& Education, Elsevier, Vol. 71, pp. 303-321.

Eryilmaz, E., Pol, J. van der, Ryan, T., Clark, P.M. and Mary, J. (2013), "Enhancing student knowledge acquisition from online learning conversations", International Journal of Computer-Supported Collaborative Learning, Springer, Vol. 8 No. 1, pp. 113-144.

Gao, F. (2013), "A case study of using a social annotation tool to support collaboratively learning", The Internet and Higher Education, Elsevier, Vol. 17, pp. 76-83.

Haslhofer, B., Sanderson, R., Simon, R., and van de Sompel, H. (2012), “Open annotations on multimedia Web resources", Multimedia Tools and Applications, Vol. 70 No. 2, pp. 847-867.

Hollett, T. and Kalir, J.H. (2017), "Mapping Playgrids for Learning Across Space, Time, and Scale”, TechTrends, Vol. 61 No. 3, pp. 236-245.

Hwang, W.Y., Shadiev, R. and Huang, S.M. (2011), "A study of a multimedia web annotation system and its effect on the EFL writing and speaking performance of junior high school students", ReCALL, Cambridge University Press, Vol. 23 No. 2 , pp. 160-180.

Jan, J.C., Chen, C.M. and Huang, P.H. (2016), "Enhancement of digital reading performance by using a novel web-based collaborative reading annotation system with two quality annotation filtering mechanisms", International Journal of Human-Computer Studies, Elsevier, Vol. 86, pp. 81-93.

Kalir, J. (in press), Social annotation enabling collaboration for open learning. Distance Education.

Li, S.C., Pow, J.W.C. and Cheung, W.C. (2015), "A delineation of the cognitive processes manifested in a social annotation environment", Journal of Computer Assisted Learning, Vol. 31 No. 1, pp. 1-13.

Lin, C.P., Chen, W., Yang, S.J., Xie, W., and Lin, C.C. (2014), "Exploring students' learning effectiveness and attitude in Group Scribbles-supported collaborative reading activities: A study in the primary classroom", Journal of Computer Assisted Learning, Vol. 30 No. 1, pp. 68-81.

Lin, J.W. and Lai, Y.C. (2013), "Harnessing collaborative annotations on online formative assessments", Journal of Educational Technology \& Society, JSTOR, Vol. 16 No. 1, pp. 263-274.

Lo, J.J., Yeh, S.W. and Sung, C.S. (2013), "Learning paragraph structure with online annotations: An interactive approach to enhancing EFL reading comprehension”, System, Elsevier, Vol. 41 No. 2, pp. 413-427.

Lu, J. and Deng, L. (2013), "Examining students' use of online annotation tools in support of argumentative reading", Australasian Journal of Educational Technology, Vol. 29 No. 2. pp. 161-171.

Marshall, C.C. (1997), "Annotation: From paper books to the digital library", Proceedings of the Second ACM International Conference on Digital Libraries - DL '97, ACM Press, New York, New York, USA, pp. 131-140.

Michaels, S., O'Connor, C. and Resnick, L.B. (2008), "Deliberative discourse idealized and realized: Accountable talk in the classroom and in civic life", Studies in Philosophy and Education, Springer, Vol. 27 No. 4, pp. $283-297$.

Miller, K., Zyto, S., Karger, D., Yoo, J. and Mazur, E. (2016), “Analysis of student engagement in an online annotation system in the context of a flipped introductory physics class", Physical Review Physics Education Research, APS, Vol. 12 No. 2 , pp. 1-12. 
Mohd Nor, N.F., Azman, H., and Hamat, A. (2013), “Investigating Students' Use of Online Annotation Tool in an Online Reading Environment", 3L: The Southeast Asian Journal of English Language Studies, Vol. 19 No. 3, pp. 87-101.

O'Hara, K. and Sellen, A. (1997), "A comparison of reading paper and on-line documents", Proceedings of the SIGCHI Conference on Human Factors in Computing Systems - CHI '97, ACM Press, New York, New York, USA, pp. 335-342.

Olson, E.D., and Brown, E. (2018), "Perceptions of students in an event management program of annotation systems and their influence on student learning", Journal of Hospitality \& Tourism Education, Taylor \& Francis, Vol. 30 No. 2, pp. 118-126.

Palinscar, A.S. and Brown, A.L. (1984), "Reciprocal teaching of comprehension-fostering and comprehension-monitoring activities", Cognition and Instruction, Vol.1 No. 2, pp.117-175.

Passig, D. and Maidel-Kravetsky, J. (2016), "The impact of collaborative online reading on summarizing skills", Education and Information Technologies, Vol. 21 No. 3, pp. 531-543.

Plevinski, J., Weible, J. and DeSchryver, M. (2017), “Anchored Annotation to Support Collaborative Knowledge Construction”, Philadelphia, PA: International Society of the Learning Sciences.

Sahota, M., Leung, B., Dowdell, S. and Velan, G.M. (2016), "Learning pathology using collaborative vs. individual annotation of whole slide images: A mixed methods trial", BMC Medical Education, BioMed Central, Vol. 16 No. 1, p. 311.

Samuel, R.D., Kim, C. and Johnson, T.E. (2011), "A study of a social annotation modeling learning system", Journal of Educational Computing Research, SAGE Publications Sage CA: Los Angeles, CA, Vol. 45 No. 1, pp. 117-137.

Su, A.Y., Yang, S. J., Hwang, W.Y. and Zhang, J. (2010), "A web 2.0-based collaborative annotation system for enhancing knowledge sharing in collaborative learning environments", Computers \& Education, Elsevier, Vol. 55 No. 2, pp. 752-766.

Sun, Y. and Gao, F. (2017), "Comparing the use of a social annotation tool and a threaded discussion forum to support online discussions", Internet and Higher Education, Vol. 32, pp. 72-79.

Thoms, J.J. and Poole, F. (2017), "Investigating linguistic, literary, and social affordances of 12 collaborative reading", Language Learning \& Technology, University of Hawaii National Foreign Language Resource Center, Vol. 21 No. 2, pp. 139-156.

Thoms, J.J., Sung, K.Y. and Poole, F. (2017), "Investigating the linguistic and pedagogical affordances of an L2 open reading environment via eComma: An exploratory study in a Chinese language course", System, Vol. 69, pp. 38-53.

Tseng, S.S., and Yeh, H.C. (2018), "Integrating reciprocal teaching in an online environment with an annotation feature to enhance low-achieving students' English reading comprehension”, Interactive Learning Environments, Vol. 26 No. 6, pp. 789-802.

van der Pol, J., Admiraal, W. and Simons, P.R.J. (2006), “The affordance of anchored discussion for the collaborative processing of academic texts", International Journal of Computer-Supported Collaborative Learning, Springer, Vol. 1 No. 3, pp. $339-357$.

Vygotsky, L. (1962), Language and Thought, MIT Press, Cambridge, MA.

W3C Web Annotation Working Group. (2016), Web Annotation Working Group, available at https://www.w3.org/annotation/.

Yang, S.J., Zhang, J., Su, A.Y. and Tsai, J.J. (2011), "A collaborative multimedia annotation tool for enhancing knowledge sharing in CSCL", Interactive Learning Environments, Taylor \& Francis, Vol. 19 No. 1, pp. 45-62.

Yang, X., Yu, S. and Sun, Z. (2013), "The effect of collaborative annotation on Chinese reading level in primary schools in China”, British Journal of Educational Technology, Vol. 44 No. 1, pp. 95-111.

Yeh, H.C., Hung, H.T. and Chiang, Y.H. (2017), "The use of online annotations in reading instruction and its impact on students' reading progress and processes", ReCALL, Cambridge University Press, Vol. 29 No. 1, pp. 22-38.

Yeh, S.W. and Lo, J.J. (2009), "Using online annotations to support error correction and corrective feedback", Computers \& Education, Elsevier, Vol. 52 No. 4, pp. 882-892.

Zarzour, H. and Sellami, M. (2017), "A linked data-based collaborative annotation system for increasing learning achievements", Educational Technology Research and Development, Vol. 65 No. 2, pp. 381-397.

Zarzour, H. and Sellami, M. (2018), "An investigation into whether learning performance can be improved by CAALDT", Innovations in Education and Teaching International, Routledge, Vol. 55 No. 6, pp. 625-632.

Zhao, N., Gao, F. and Yang, D. (2018), "Examining student learning and perceptions in social annotation-based translation activities", Interactive Learning Environments, Routledge, Vol. 26 No. 7, pp. 958-969. 